\title{
Avaliação ecocardiográfica em pessoas vivendo com o vírus da imunodeficiência humana: revisão narrativa
}

\author{
Valuation of echocardiography in people living with the Human Immunodeficiency Virus: a \\ narrative review
}

\section{Evaluación ecocardiográfica en personas que viven con el virus de la inmunodeficiencia humana: revisión narrativa}

Kelly Simone Castro dos Santos ${ }^{1,2 *}$, Marlucia do Nascimento Nobre ${ }^{1,2}$, Anne Elizabeth Andrade Sadala Marques ${ }^{3}$, Aline Brasil Aranha ${ }^{2,3}$, Andreza Araújo de Oliveira ${ }^{5}$, Suhã Ono Santos ${ }^{5}$, Vanine de Lourdes Aguiar Lima Fragoso ${ }^{6}$, Antônio Augusto de Souza Marinho ${ }^{7}$, Marcelo Mouco Fernandes $^{8,9}$, João Marcos Barbosa Ferreira ${ }^{4,5}$.

\section{RESUMO}

Objetivo: Abordar as manifestações ecocardiográficas de Doenças Cardiovasculares (DCV) na infecção pelo Vírus da Imunodeficiência Humana (HIV), com ênfase nos dados que contribuem para o diagnóstico precoce e antecipação de complicações. Revisão bibliográfica: Atualmente, com a moderna Terapia Antirretroviral (TARV) e prolongamento da expectativa de vida da Pessoa Vivendo com HIV (PVHIV) com carga viral controlada, a atenção e o cuidado têm sido direcionados ao combate de doenças crônicas e complicações secundárias, não infecciosas. As disfunções cardíacas nos pacientes portadores de HIV ocorrem com frequência variável e diversidade de apresentações clínicas e constituem uma das causas mais comuns de morte nesta população. A complexa e multifatorial patogênese das complicações cardiovasculares associadas ao HIV envolve fatores de risco tradicionais para DCV, efeitos deletérios do vírus, status inflamatório e distúrbios metabólicos relacionados a TARV. A maioria das alterações detectadas pelo ecocardiograma persistem durante o acompanhamento, de forma assintomática, sugerindo um longo período de alterações cardíacas subclínicas. Considerações finais: A ecocardiografia demonstra ser uma importante ferramenta no seguimento de pacientes com HIV para diagnóstico primário e detecção precoce do acometimento cardiovascular, permitindo o tratamento em tempo oportuno e melhora da qualidade e expectativa de vida dos pacientes.

Palavras-chave: Doença Cardiovascular (DCV), Vírus da Imunodeficiência Humana (HIV), Terapia Antirretroviral (TARV), Ecocardiografia, Risco cardiovascular.

\footnotetext{
ABSTRACT

Objective: To address the echocardiographic manifestations of Cardiovascular Diseases (CVD) in people living with Human Immunodeficiency Virus (HIV) infection, with an emphasis on data that contribute to both early diagnosis and anticipation of complications. Bibliographic review: Currently, with modern Antiretroviral Therapy (ART) and prolongation of life expectancy of people living with HIV (PLHIV) with controlled HIV viral load, attention and care have been directed to combat chronic diseases and secondary complications, not infectious. Cardiac dysfunction in patients with HIV occur with variable frequency and diversity of clinical presentations and is one of the most common reasons for death in this population. The complex and multifactorial pathogenesis of HIV-associated cardiovascular complications involves traditional risk factors for

1 Universidade Federal do Amazonas (UFAM), Manaus - AM. *E-mail: dra.kellysimone.cardio@gmail.com

${ }^{2}$ Hospital Universitário Getúlio Vargas (HUGV), Manaus - AM.

${ }^{3}$ Fundação Hospital do Coração Francisca Mendes (HUFM), Manaus - AM.

${ }^{4}$ Universidade do Estado do Amazonas (UEA), Manaus - AM.

5 Universidade Nilton Lins (UNL), Manaus - AM.

${ }^{6}$ Fundação de Medicina Tropical Dr. Heitor Vieira Dourado, Manaus - AM.

${ }^{7}$ Hospital Adventista de Manaus (HAM), Manaus - AM.

${ }^{8}$ Pulsar Clínica Cardiológica, Manaus - AM.

${ }^{9}$ Instituto de Mamas da Amazônia, Manaus - AM.
} 
CVD, deleterious effects of the virus, inflammatory status, and ART-related metabolic disturbances. Most of the changes detected by echocardiography remain asymptomatic during follow-up, suggesting a long period of subclinical cardiac changes. Final consideration: Echocardiography proves to be an important tool in the follow-up of patients with HIV for primary diagnosis and early detection of cardiovascular involvement, enabling timely treatment and improving the quality and life expectancy of patients.

Keywords: Cardiovascular Disease (CVD), Human Immunodeficiency Virus (HIV), Antiretroviral Therapy (ART), Echocardiography, Cardiovascular risk.

\section{RESUMEN}

Objetivo: Abordar las manifestaciones ecocardiográficas de Enfermedades Cardiovasculares (ECV) en la infección por el Virus de la Inmunodeficiencia Humana (VIH), con énfasis en los datos que contribuyen al diagnóstico precoz y anticipación de complicaciones. Revisión bibliográfica: Actualmente, con la moderna Terapia Antirretroviral (TARV) y prolongación de la expectativa de Vida de la Persona Viviendo con VIH con carga viral controlada, la atención y el cuidado han sido dirigidos al combate de enfermedades crónicas y complicaciones secundarias, no infecciosas. Las disfunciones cardíacas en pacientes con VIH ocurren con frecuencia variable y diversidad de presentaciones clínicas y constituyen una de las causas más comunes de muerte en esta población. La patogénesis compleja y multifactorial de las complicaciones cardiovasculares asociadas al VIH implica factores de riesgo tradicionales para ECV, efectos nocivos del virus, estado inflamatorio y trastornos metabólicos relacionados con TARV. La mayoría de las alteraciones detectadas por el ecocardiograma persisten durante el seguimiento, de forma asintomática, sugiriendo un largo período de alteraciones cardíacas subclínicas. Consideraciones finales: La ecocardiografía demuestra ser una importante herramienta en el seguimiento de pacientes con VIH para diagnóstico primario y detección precoz del acometimiento cardiovascular, permitiendo el tratamiento en tiempo oportuno y mejorando la calidad y expectativa de vida de los pacientes.

Palabras clave: Enfermedades Cardiovasculares (ECV), Virus de la Inmunodeficiencia Humana (VIH), Terapia Antirretroviral (TARV), Ecocardiografía, Riesgo cardiovascular.

\section{INTRODUÇÃO}

A Organização Mundial de Saúde (OMS) estima que, em 2020, a infecção pelo Vírus da Imunodeficiência Humana (HIV) tenha acometido 37,6 milhões de pessoas no mundo e, em 2021, 690 mil de pessoas morreram de doenças relacionadas à Síndrome da Imunodeficiência Adquirida (SIDA) (UNITED NATIONS PROGRAMME ON HIV/AIDS (UNAIDS), 2021).

No Brasil, no período de 1980 a 2020, o número de casos de SIDA notificados pelo Sistema de Informação de Agravos de Notificação (SINAN) foi de 1.011.617, com predomínio do sexo masculino e faixa etária de 20 a 39 anos (MINISTÉRIO DA SAÚDE, 2021).

A infecção pelo HIV, até então apontada como uma condição progressiva e fatal, passou a ser considerada, após o emprego mais difundido, precoce e eficaz da Terapia Antirretroviral (TARV), como uma doença crônica gerenciável, marcada pelo elevado risco de Doenças Cardiovasculares (DCVs) (KUMAR A, et al., 2021).

As manifestações cardiovasculares são as mais diversas, consequentes à própria infecção viral, à autoimunidade, à reação imunológica diante de outras infecções, à inflamação crônica, a neoplasias, à imunossupressão prolongada, à desnutrição e à cardiotoxicidade dos medicamentos (ARSHAD A, et al., 2000).

As taxas de complicações cardiovasculares como acidente vascular encefálico, infarto agudo do miocárdio, insuficiência cardíaca e outras apresentações de DCV como hipertensão pulmonar e morte súbita, são significativamente maiores em Pessoas que Vivem com o HIV (PVHIV), mesmo em indivíduos com carga viral controlada, do que em indivíduos sem a infecção viral (MATTHEW JF, et al., 2019).

Diante do exposto, o objetivo do estudo foi discutir as diversas formas de manifestação cardíaca em PVHIV, com ampla revisão da literatura, destacando a importância do ecocardiograma transtorácico ao longo do seguimento clínico dos pacientes. 


\section{REVISÃO BIBLIOGRÁFICA}

\section{História da pandemia do HIV}

Há quarenta anos a humanidade se deparava com os primeiros casos de uma doença causada por um vírus, um marco na história da saúde mundial. Esse vírus, identificado como Vírus da Imunodeficiência Humana (do inglês HIV), é um retrovírus, da subfamília dos Lentiviridae, que afeta células específicas do sistema imunológico, os linfócitos T CD4 (LT CD4+), alterando seu DNA, passando ali, a se replicar. A progressão da doença já está bem documentada e a não intervenção culmina com a SIDA (GUTIERREZ EB, et al., 2013).

A infecção pelo HIV cursa com amplo espectro de apresentações clínicas. Com LT CD4+ em níveis acima de 350 células $/ \mathrm{mm}^{3}$, infecções bacterianas podem se manifestar. Com a progressão da doença e redução dos níveis de $L T$ CD4+, a imunossupressão é marcada pelas infecções virais, fúngicas e bacterianas (MINISTÉRIO DA SAÚDE, 2013).

Nessa fase, o próprio vírus pode causar doenças a partir da ação direta a certos órgãos ou por processos inflamatórios, tais como miocardiopatia, nefropatia e neuropatias, que podem estar presentes durante toda a evolução da infecção pelo HIV, de acordo com as informações verificadas nos dados epidemiológicos fornecido pelo Ministério da Saúde em seu portal do SINAN (MINISTÉRIO DA SAÚDE, 2019).

Desde 1996, o Brasil distribui gratuitamente pelo Sistema Único de Saúde (SUS) medicamentos para o tratamento do HIV e, desde 2013, o Ministério da Saúde tem ampliado essa oferta às populações-chave e prioritárias para o HIV, de acordo com o relatório de monitoramento clínico do HIV de 2019 (MINISTÉRIO DA SAÚDE, 2019).

A partir de então, as PVHIV que receberam a TARV passam a ter uma longa expectativa de vida e a SIDA a ter uma abordagem diferente passando a ser conduzida como uma doença crônica (KUMAR A, et al., 2021). Dados do Centro de Controle de Doenças (CDC) indicam que a mortalidade devido ao HIV caiu de uma taxa de mortalidade anual ajustada por idade de 17 por 1000 em 1995 para 2 por 1000 em 2014 (CDC, 2021).

O acometimento cardíaco no HIV foi inicialmente descrito em 1983, a partir do diagnóstico de sarcoma de Kaposi miocárdico em paciente com SIDA (AUTRAN BR, et al., 1983). Estudos de autopsia realizados na era pré-TARV demonstram prevalências de $28 \%$ a $73 \%$ de acometimento cardíaco no PVHIV, com envolvimento de vasos, endocárdio, miocárdio e pericárdio (ARSHAD A, et al., 2000).

Estudos adicionais são necessários a fim de que se conheça melhor os mecanismos e possíveis impactos na morbimortalidade dos pacientes com HIV, visto que em 2030, $73 \%$ das pessoas com HIV terão 50 anos ou mais e 78\% terão doença cardiovascular (BLOOMFIELD GS, et al., 2017).

\section{Monitoramento cardiovascular}

A triagem e monitoramento de PVHIV são essenciais para detecção e intervenção precoces, uma vez que somente o exame físico pode não ser tão fidedigno em pacientes imunocomprometidos. O diagnóstico tardio favorece a continuidade da cadeia de transmissão do vírus e dificulta a recuperação imunológica do indivíduo (BLOOMFIELD GS, et al, 2017).

As alterações cardíacas em PVHIV apresentam frequência variável e diversidade de manifestações, em sua maioria, subclínicas. O exame ecocardiográfico é capaz de identificar condições comuns e que se relacionam a pior prognostico como endocardite, valvopatia e derrame pericárdico, além de identificar trombos e massas no miocárdio, como sarcoma e linfoma, não tão raros em PVHIV (NAHASS R, et al., 2011).

A ecocardiografia pode identificar os PVHIV com elevado risco de acometimento cardíaco, porém o significado clínico dessas alterações estruturais permanece desconhecido (LUMSDEN RH e BLOOMFIELD GS, 2016). É o melhor exame custo-efetivo em pacientes com cardiomiopatia e pode auxiliar na identificação da causa dos sintomas apresentados, bem como na diferenciação de alterações secundárias a doença valvar, pericárdica, infiltrativa, isquêmica ou defeitos congênitos (NAHASS et AL, 2011).

Os principais achados ecocardiográficos em pessoas vivendo com o HIV são: disfunção sistólica dos ventrículos direito e esquerdo, disfunção diastólica do ventrículo esquerdo (VE) e hipertensão pulmonar (ERDOL M, et al., 2021). 


\section{Disfunção sistólica do ventrículo esquerdo e HIV}

Pacientes que não iniciaram a TARV e com baixas contagens de CD4+ têm maior incidência de coinfecções, inflamação sistêmica e coagulopatia que podem ser associadas à cardiomiopatia isquêmica ou não isquêmica (SUKRU C, et al., 2018). A baixa contagem de células T e alta carga viral são os principais indicadores no desenvolvimento de insuficiência cardíaca em PVHIV (FREIBERG MS, et al., 2017).

O diagnóstico da cardiomiopatia dilatada associada à infecção pelo HIV é clínico e ecocardiográfico. $O$ dano miocárdico leva à disfunção sistólica do Ventrículo Esquerdo (VE), hipocinesia difusa, dilatação global das câmaras, aumento da massa ventricular esquerda que podem ser observados pela ecocardiografia transtorácica. Fração de ejeção reduzida e aumento da espessura ventricular têm sido associadas a maior mortalidade (BLOOMFIELD G, et al., 2017).

Ao longo das décadas de 1980 e 1990, antes da disponibilidade da TARV, a presença de insuficiência cardíaca em PVHIV ocorreu principalmente no contexto de miocardite relacionada aos efeitos diretos do vírus, infecções oportunistas, deficiências nutricionais ou imunossupressão severa. A miocardite é particularmente comum nos estágios finais da infecção pelo HIV. Alta incidência de miocardite está associada a contagem de CD4+ inferior a 400 células/mm (BLOOMFIELD G, et al., 2017).

A cardiopatia associada ao HIV era caracterizada como disfunção sistólica sintomática associada a um ventrículo esquerdo dilatado e baixa sobrevida. Atualmente as manifestações cardiovasculares associadas ao HIV incluem insuficiência cardíaca sintomática com disfunção ventricular esquerda com ou sem dilatação ventricular, qualquer comprometimento sistólico ou disfunção diastólica em pacientes com HIV assintomáticos (LUMSDEN RH e BLOOMFIELD GS, 2016).

A redução de infecções oportunistas em pacientes em TARV pode ser responsável pela queda na incidência de miocardite, embora o uso de regimes baseados em zidovudina (AZT) possa estar associado a maior risco de cardiomiopatia, por dano mitocondrial e necrose miocárdica que pode ser reversível com a interrupção da medicação (LUMSDEN RH e BLOOMFIELD GS, 2016).

\section{Disfunção diastólica do ventrículo esquerdo e HIV}

O fenótipo do acometimento cardiovascular do HIV tem se modificado gradualmente, de disfunção sistólica do VE para acometimento mais comum da função diastólica isoladamente. Através de alguns estudos se evidenciou que PVHIV apresentam uma maior prevalência de disfunção diastólica e em idade mais jovem que pacientes HIV negativos (LUMSDEN RH e BLOOMFIELD GS, 2016).

A disfunção diastólica é um fator de risco para insuficiência cardíaca de fração de ejeção preservada, mortalidade cardiovascular e mortalidade por todas as causas (BLOOMFIELD GS, et al, 2017). Ademais, pode estar presente em torno de $26 \%$ a $50 \%$ de PVHIV, número que é aproximadamente 10 vezes maior que na população geral na mesma faixa etária (BUTLER J, et al., 2018).

O subestudo cardiovascular do Multicenter AIDS Cohort Study (MACS), que arrolou 721 homens avaliados por tomografia computadorizada cardíaca, evidenciou que os homens com HIV possuíam massa ventricular esquerda significativamente maior que os homens sem HIV. A Hipertrofia Ventricular Esquerda (HVE) está relacionada com aumento do risco de disfunção diastólica do VE e consequentemente de fibrilação atrial e insuficiência cardíaca (HUTCHINS E, et al., 2019).

Os mecanismos envolvidos ainda não são inteiramente conhecidos, mas as evidências atuais sugerem que os processos imunológicos, inflamação crônica com um maior depósito de colágeno e fibrose miocárdica, têm papel central na patogênese da disfunção diastólica em PVHIV, bem como a TARV parece estar envolvida (HUTCHINS E, 2019).

Em geral, a disfunção diastólica é precedida por aumento da massa ventricular esquerda, hipertrofia ventricular esquerda, aumento das pressões de enchimento que leva ao aumento da pressão arterial pulmonar, achados comuns em pacientes com HIV (HUTCHINS E, 2019).

O exame ecocardiográfico pode detectar disfunção diastólica isolada em pacientes em fase assintomática. Ecocardiografia permanece o padrão para detecção de disfunção sistólica ventricular, além de disfunção diastólica e tensão miocárdica anormal, que são frequentemente as únicas anormalidades ecocardiográficas encontradas em PVHIV assintomáticos em TARV (LUMSDEN RH e BLOOMFIELD GS, 2016). 


\section{Hipertensão pulmonar e HIV}

A Hipertensão Pulmonar (HP) é uma doença progressiva caracterizada por elevação da pressão arterial pulmonar e Resistência Vascular Pulmonar (RVP), levando à insuficiência Ventricular Direita (VD) e morte prematura (ARAÚJO I, et al., 2014).

Embora a HP seja uma condição rara, a prevalência em PVHIV é alta e considerada devastadora, com o prognóstico reservado e piores taxas de sobrevivência (KUMAR A, et al., 2021), estimada em aproximadamente $0,5 \%$ antes da terapia TARV, mas indicando que o emprego de fármacos antirretrovirais não teve um grande impacto na prevenção da HP pelo HIV (ERDOL MA, et al., 2021).

Os fatores responsáveis pelas complicações pulmonares crônicas no paciente com HIV são condições inflamatórias persistentes, desequilíbrio do sistema imunológico e estresse oxidativo que ocasionam disfunção endotelial (KUMAR A, et al., 2021).

Várias proteínas do HIV, como Tat, Nef e gp120, ocasionam atividade inflamatória vascular e desregulação da função do endotélio e do músculo liso induzindo ao fenótipo suscetível a HP. Ainda é frequente o uso de drogas ilícitas e exposição a coinfecções em PVHIV, o que aumenta a capacidade do vírus de induzir lesão vascular e consequente HAP. Outras condições como disfunção cardíaca esquerda e doença parenquimatosa pulmonar também favorecem o desenvolvimento e exacerbação da doença vascular pulmonar (KUMAR A, et al., 2021).

Altas cargas virais de HIV ( $>500$ cópias / mL) e baixas contagens de células CD4 ( $<200$ células / $\mu$ ) predispõe pressão sistólica da artéria pulmonar (PSAP) excedendo $40 \mathrm{mmHg}$. Mesmo com valores PSAP considerados normais e bem abaixo do limite no qual a avaliação invasiva é recomendada, o risco de mortalidade é aumentado em PVHIV, mesmo na ausência de outras comorbidades, sendo os indivíduos negros particularmente mais vulneráveis. Esses achados justificam reconsideração das abordagens atuais para o diagnóstico de HP e recomendações futuras para rastreamento e vigilância da hipertensão pulmonar em pacientes com HIV (BRITTAIN EL, et al., 2018).

A maioria dos pacientes com HIV são diagnosticados com HP principalmente nos estágios finais da doença. $O$ tempo médio do diagnóstico até a morte nesses pacientes é de aproximadamente 6 meses (ERDOL MA, et al., 2021). A detecção precoce de HP é essencial, pois poderia ser fornecido tratamento em tempo oportuno, com redução dos sintomas e melhor expectativa de vida dos pacientes (SCHWARZEZANDER C, et al., 2015).

A ecocardiografia constitui a ferramenta para diagnóstico primário e detecção de HP em pacientes com HIV. Na triagem ecocardiográfica de um estudo prospectivo, a HP foi detectada em $6,1 \%$ de pacientes infectados pelo HIV. Destes, $13 \%$ apresentaram sintomas de dispneia e fadiga, e o diagnóstico de HP foi confirmado por cateterismo cardíaco direito. Pacientes com PSAP $>30 \mathrm{mmHg}$ tinham maior probabilidade de ser do sexo feminino e de ter histórico de uso de drogas injetáveis (SCHWARZE-ZANDER C, et al., 2015).

As estimativas ecocardiográficas de PSAP são particularmente relevantes em áreas endêmicas onde os laboratórios de cateterização são raros e a projeção de risco é necessariamente baseada em dados ecocardiográficos (BRITTAIN E, et al., 2018).

Um marcador ecocardiográfico, não invasivo e derivado de Doppler simples, é o tempo de trânsito do pulso pulmonar ( $\mathrm{PPTT}$ ) usado para avaliar a rigidez arterial pulmonar e preditor da doença vascular em pacientes com HIV. Com o aumento da rigidez e da pressão arterial, a Velocidade da Onda de Pulso (VOP) aumenta, enquanto o pPTT, excursão sistólica de pico anular tricúspide (TAPSE) e alteração da Variação da Área Fracionada (FAC) do ventrículo direito são significativamente menores em pacientes com HIV comparado a pacientes sem infecção (ERDOL MA, et al., 2021).

O PPTT parece ter o potencial de ser um melhor preditor prognóstico de doença vascular pulmonar em pacientes com HIV do que a Resistência Vascular Pulmonar (RVP), mesmo quando a pressão arterial pulmonar ainda está dentro da normalidade (ERDOL MA, et al., 2021). 


\section{Disfunção ventricular direita e HIV}

O envolvimento cardíaco relacionado ao HIV é representado por disfunção assintomática do ventrículo direito (detectável apenas por ecocardiografia avançada), HP e disfunção do ventrículo esquerdo (DEIDDA M, et al., 2018).

Vários estudos foram realizados para avaliar a função do VD em PVHIV e incluíram parâmetros tradicionais da função do VD, como excursão sistólica de pico anular tricúspide (TAPSE) e índice de desempenho miocárdico (SCHWARZE-ZANDER C, et al, 2015).

Erdol MA, et al. (2021), demonstraram que os parâmetros associados à disfunção do VD (pPTT, TAPSE, FAC do VD) foram estatisticamente mais baixos em pacientes com HIV. Na disfunção do VD, a pressão do Átrio Direito (AD) aumenta. Consequentemente, o gradiente AD-VD diminui e a PSAP pode ser subestimada como na regurgitação tricúspide grave.

Desta forma, mudanças significativas em pTT, TAPSE e FAC não são observadas no parâmetro PSAP. A FAC é um método relativamente simples para avaliar a função do VD e pode ser mais valioso que o TAPSE na avaliação das funções do VD. Este estudo mostrou que os pacientes com HIV tinham o pPTT mais curto e sua correlação com o TAPSE. O pPTT foi positivamente correlacionado com a FAC do ventrículo direito (ERDOL MA, et al., 2021).

\section{Strain bidimensional em pacientes vivendo com HIV}

Em alguns pacientes, mesmo quando o ecocardiograma convencional realizado na rotina é normal, pode ser encontrada disfunção incipiente através da análise da deformidade miocárdica pelo strain bidimensional. A técnica de strain bidimensional avalia a função cardíaca através da medida da porcentagem de mudança da distância entre dois pontos do miocárdio durante a sístole. A média feita nos 17 segmentos do ventrículo esquerdo representa o Strain Global Longitudinal (SGL) que é a medida mais utilizada na prática clínica e em publicações científicas. Desta forma é avaliada a deformidade miocárdica que pode estar alterada de forma precoce mesmo antes de surgir redução da fração de ejeção em PVHIV (MCCRAY AW, et al.,2020).

Várias publicações demonstraram a presença de redução do SGL em PVHIV. Alenezi F, et al. (2019) encontraram redução do SGL abaixo do valor da normalidade em cerca de 30\% destes pacientes. Outros estudos, apesar de apresentarem medidas de SGL ainda dentro dos valores da normalidade, apresentaram diferença estatisticamente significativa entre o grupo de PVHIV quando comparados com um grupo controle de pessoas saudáveis (MCCRAY AW, et al., 2020).

Estas alterações encontradas no strain bidimensional ocorreram em vários subgrupos de PVHIV. A maioria dos autores estudou crianças, adolescentes e jovens adultos (CAPOTOSTO L, et al., 2019). Com relação ao uso da TARV, a maioria demonstrou redução do GLS em pacientes em uso desta terapia (CINCIN A, et al., 2020).

Porém, mesmo pacientes com diagnóstico de infecção por HIV recente e ainda sem uso de TARV apresentam evidência de disfunção ventricular através do strain bidimensional. Karavidas A, et al. (2015) avaliou 41 pacientes infectados pelo HIV virgens de tratamento e comparou com 20 pacientes de um grupo controle pareado por sexo e idade.

Todas as medidas de strain foram menores no grupo com HIV, comparado com o controle. Além disto, estas alterações do strain bidimensional ocorrem mesmo em pacientes totalmente livres de outros fatores de risco cardiovasculares ou outras comorbidades como demonstrado por Deidda $\mathrm{M}$, et al. (2018). Isto sugere que estas alterações são independentes de outros fatores e decorrem exclusivamente de aspectos próprios da infecção pelo HIV ou seu tratamento.

Alguns fatores são sugeridos como mecanismos e causas da disfunção miocárdica detectada pelo strain. Stobe S, et al. (2021) relataram um caso de miocardite aguda pelo vírus HIV com alteração da deformação miocárdica, sugerindo papel da presença do vírus no miocárdio como possível fator desencadeante.

Alenezi F, et al. (2019) avaliaram o status inflamatório e encontrou correlação entre baixos níveis de CD4 e nadir de CD4 com piores medidas de SGL, sugerindo que o perfil inflamatório também é importante no mecanismo da disfunção miocárdica. Outro possível mecanismo etiológico para a disfunção miocárdica é o grupo de medicamento utilizado na TARV. 
Cincin A, et al. (2020) demonstraram piores níveis de SGL em pacientes utilizando inibidores da protease e Inibidores da Transcriptase Reversa Não Nucleosídeos (ITRNN), sugerindo que a classe de medicamento utilizado também tem papel importante no desenvolvimento de disfunção cardíaca.

\section{Alterações ecocardiográficas em crianças vivendo com HIV}

Estudos conduzidos na era pré-TARV revelaram prevalência de 70 a 90\% de disfunções cardíacas entre crianças infectadas pelo HIV (LUGINBUHL LM, et al., 1993). Mas apesar do uso da terapia combinada altamente eficaz nas últimas décadas, tais disfunções continuam sendo descritas nessa população, com frequência variável, e diversidade de apresentações clínicas, em sua maioria, subclínicas (MAJONGA ED, et al, 2018).

Starc T, et al. (2002) recomendaram que estes pacientes fossem submetidos ao ecocardiograma logo após o diagnóstico da infecção pelo HIV, devendo este ser repetidos a cada dois anos em pacientes assintomáticos, ou anualmente quando apresentassem sintomas de insuficiência cardíaca, doença respiratória inexplicável ou infecção sintomática pelo próprio vírus.

Um estudo em Jakarta, Indonésia, acompanhou 114 crianças com transmissão vertical, sendo 56 virgens de TARV e 58 expostas a TARV, e um grupo controle de 51 crianças saudáveis, quando mostrou que as crianças infectadas com HIV, virgens de TARV, apresentavam uma diminuição substancial da função sistólica cardíaca, enquanto as expostas à TARV apresentavam paredes ventriculares mais espessas com diâmetro interno maior e maior massa, porém, menor comprometimento funcional (IDRIS NS, et al., 2016).

O estudo CHAART-1 mostrou que a exposição a TARV intraútero e no período pós-natal imediato associou-se à redução da massa do ventrículo esquerdo e menor espessura da parede septal, além de comprometimento no crescimento cardíaco, ao comparar ecocardiogramas de filhos de mães infectadas pelo HIV, quando um grupo foi exposto a TARV e outro não (LIPSHULTZ SE, et al., 2011).

No Brasil, antes da TARV ser indicada para todas as crianças infectadas a partir do diagnóstico, Cunha M, et al. (2008), avaliaram 93 crianças por meio da realização de ecocardiograma, quando encontraram uma prevalência de $43 \%$ de alterações cardíacas, detectando também uma associação positiva entre a ausência do uso de terapia tríplice e presença de disfunção ventricular esquerda.

Vallilo NG, et al. (2020) realizaram avaliação ecocardiográfica seriada de uma coorte de crianças infectadas verticalmente pelo HIV na era pós-TARV e identificaram a dilatação de VE como sendo a alteração mais comum nesta população (21,6\%), tendo sido transitória em 14 das 32 crianças diagnosticadas. A dilatação de VD foi encontrada em $18,9 \%$ da população, 53,5\% transitórias, associadas neste estudo a categoria clínica C, ao uso dos ITRNN, infecções oportunistas e LIP (VALLILO NG, et al., 2020).

Majonga ED, et al. (2018), avaliaram 201 crianças com idade entre 6 e 16 anos e encontraram alterações no ecocardiograma de $42 \%$ delas, tendo sido a disfunção ventricular diastólica esquerda o achado mais comum (23\%), seguido da hipertrofia de ventrículo esquerdo (11\%). Esse estudo também associou o uso da nevirapina ao achado de hipertrofia do ventrículo esquerdo. Essa população foi seguida em uma coorte de 18 meses e observou-se que apesar do uso da TARV, apresentaram maior risco de desenvolver dilatação do ventrículo direito, sem associação a disfunção pulmonar.

A maioria das alterações detectadas persistiram durante o acompanhamento, de forma assintomática, sugerindo um longo período de alterações cardíacas subclínicas. As alterações transitórias, disfunção sistólica do VE e hipertrofia de VE foram associadas a quadros de miocardite infecciosa aguda (MAJONGA ED, et al., 2020).

\section{CONSIDERAÇÕES FINAIS}

Embora na última década tenha ocorrido grande progresso, existem lacunas consideráveis na compreensão da DCV associada ao HIV. Vários estudos analisaram a fisiopatologia da disfunção cardíaca no HIV, mas relativamente poucos foram dedicados às manifestações ecocardiográficas nesta população. $A$ ecocardiografia demonstra ser uma importante ferramenta para diagnóstico primário, detecção precoce e seguimento do acometimento cardiovascular em pacientes com HIV. Estudos futuros devem abordar as lacunas na implementação de dados ecocardiográficos, para garantir a identificação e tratamento precoce de pessoas com risco de DCV ou com DCV existentes. 


\section{REFERÊNCIAS}

1. ALENEZI F, et al. Global Longitudinal Strain and Immune Status in Patients Living With Human Immunodeficiency Virus. Am. J. Cardiol., 2019; 124(6): 966-971.

2. ARAÚJO I, et al. Hipertensão arterial pulmonar relacionada à infecção pelo vírus da imunodeficiência humana: uma série de casos. World J. Cardiol., 2014; 6:495-501.

3. ARSHAD A, et al. Cardiac complications of human immunodeficiency virus infection: diagnostic and therapeutic considerations. Heart Disease, 2000; 2:133-45.

4. AUTRAN B, et al. AIDS in a Haitian woman with cardiac Kaposi's sarcoma and Whipple's disease. Lancet, 1983; I: 76 78.

5. BLOOMFIELD G, et al. Longitudinal evolution of cardiac structure and function in patients living with human immunodeficiency virus in the antiretroviral therapy era. Circulation, 2017; 136: A19287

6. BRITTAIN E, et al. Increased echocardiographic pulmonary pressure in HIV-infected and -uninfected individuals in the veterans aging cohort study. American Journal of Respiratory and Critical Care Medicin, 2018; 197: 923-932

7. BUTLER J, et al. Diastolic Dysfunction in Individuals With Human Immunodeficiency Virus Infection: Literature Review, Rationale and Design of the Characterizing Heart Function on Antiretroviral Therapy (CHART) Study. J. Card. Fail., 2018; 24(4): 255-265.

8. CAPOTOSTO L, et al. Assessment of Biventricular Function by Three-Dimensional Speckle Tracking Echocardiography in Adolescents and Young Adults with Human Immunodeficiency Virus Infection: A Pilot Study. Cardiology (Switzerland), 2019; 144(3-4): 101-111.

9. CENTRO DE CONTROLE DE DOENÇAS (CDC). HIV Estatistics Center. 2021. Disponivel em: https://www.cdc.gov/hiv/statistics/index.html. Acessado em: 15 set. 2021.

10. CINCIN A, et al. Ventricular and atrial functions assessed by speckle-tracking echocardiography in patients with human immunodeficiency virus. Journal of Clinical Ultrasound, 2021; 49(4): 341-350

11. CRAY M, et al. Early Cardiac Dysfunction in Children and Young Adults with Perinatally Acquired HIV. AIDS, 2020; 34(4): 539-548.

12. CUNHA M, et al. AIDS na infância: acometimento cardíaco com e sem a terapia antirretroviral tríplice combinada. Arq. Bras. Cardiol., 2008; 90(1):11-7.

13. DEIDDA M, et al. Early Right Ventricular Dysfunction in Highly Selected (Totally Free from Cardiovascular Risk Factors and Other Comorbidities) Human Immunodeficiency Virus Patients: A Pilot Study with Advanced Echocardiography, J Cardiovasc Echogr, 2018; 28(4): 228-232.

14. ERDOL M, et al. Assessment of Pulmonary Arterial Hemodynamic and Vascular Changes by Pulmonary Pulse Transit Time in Patients with Human Immunodeficiency Virus Infection. J. Cardiovasc. Echogr., 2021; 31(1):6-10.

15. FEINSTEIN M, et al. Características, prevenção e tratamento de doenças cardiovasculares em pessoas que vivem com HIV: uma declaração científica da American Heart Association. Circulation, 2019; Author manuscript; available in PMC 2021 Mar 25. Published in final edited form as: Circulation. 2019 Jul 9; 140(2): e98-e124.

16. FREIBERG MS, et al. Association between HIV infection and the risk of heart failure with reduced ejection fraction and preserved ejection fraction in the antiretroviral therapy era: results from the veterans aging cohort study. JAMA Cardiol., 2017; 2(5): 536-546.

17. GUTIERREZ EB, et al. Retroviroses e Síndrome da Imunodeficiência Adquirida in: Dinâmica das doenças infecciosas e parasitárias. 2a ed. Rio de Janeiro: Guanabara Koogan, 2013; 165; 2080 p.

18. HUTCHINS E, et al. HIV Infection Is Associated with Greater Left Ventricular Mass in the Multicenter AIDS Cohort Study. AIDS Res Hum Retroviruses, 2019; 35(8):755-761.

19. IDRIS S, et al. Cardiac effects of antiretroviral-naÃ ve versus antiretroviral-exposed HIV infection in children. PLoS One, 2016; 11(1).

20. KARAVIDAS A, et al. Myocardial deformation imaging unmasks subtle left ventricular systolic dysfunction in asymptomatic and treatment-naïve HIV patients. Clinical Research in Cardiology, 2015; 104(11): 975-981.

21. KUMAR A, et al. Impact of human immunodeficiency virus on pulmonary vascular disease. Global Cardiology Science and Practice, 2021; 12.

22. LIPSHULTZ S, et al. Cardiac effects of antiretroviral therapy in HIV-negative infants born to HIV-positive mothers: NHLBI CHAART-1 (National Heart, Lung, and Blood Institute Cardiovascular Status of HAART Therapy in HIV-Exposed Infants and Children Cohort Study). J. Am. Coll. Cardiol., 2011; 57(1): 76-85.

23. LUGINBUHL LM, et al. Cardiac Morbidity and Related Mortality in Children With HIV Infection. JAMA J. Am. Med. Assoc., 1993; 269(22): 2869-75.

24. LUMSDEN RH e BLOOMFIELD GS. The Causes of HIV-Associated Cardiomyopathy: A Tale of Tworlds. BioMed Research International, 2016.

25. MAJONGA ED, et al. High prevalence of echocardiographic abnormalities in older HIV-infected children taking antiretroviral therapy. AIDS. 2018; 32(18): 2739-48. 
26. MAJONGA E, et al. Incidence and progression of echocardiographic abnormalities in older children with human immunodeficiency virus and adolescents taking antiretroviral therapy: A prospective cohort study. Clin Infect Dis. 2020; 70(7): 1372-8.

27. MINISTÉRIO DA SAÚDE (MS). Sistema de Informação de Agravos de Notificação. Dados epidemiológicos do SINAN. Aids - desde 1980. 2021. Disponível em: http://portalsinan.saude.gov.br/dados-epidemiologicos-sinan\# . Acessado em: 1 set. 2021.

28. MINISTÉRIO DA SAÚDE (MS). Departamento de doenças de condições crônicas e infeções sexualmente transmissíveis. Protocolo clínico e diretrizes terapêuticas para manejo da infecção pelo HIV em adultos. 2013. Disponível em: http://www.aids.gov.br/pt-br/pub/2013/protocolo-clinico-e-diretrizes-terapeuticas-para-manejo-dainfeccao-pelo-hiv-em-adultos. Acessado em: 5 ago. 2021.

29. MINISTÉRIO DA SAÚDE (MS). Departamento de doenças de condições crônicas e infeções sexualmente transmissíveis. Relatório de monitoramento clínico do HIV. 2019. Disponível em: http://www.aids.gov.br/system/tdf/pub/2016/67063/relatorio_de_monitoramento_clinico2.pdf?file=1\&type=node\&id=6 7063\&force=1. Acessado em: 10 ago. 2021.

30. NAHASS R, et al. Infective endocarditis in intravenous drug users: a comparison of human immunodeficiency virus type 1-negative and -positive patients. J. Infect. Dis. 1990; 162: 967-970.

31. SCHWARZE-ZANDER C, et al. Hipertensão pulmonar na infecção por HIV: um estudo ecocardiográfico prospectivo. HIV Med., 2015; 16: 578-82.

32. STARC T, et al. Incidence of cardiac abnormalities in children with human immunodeficiency virus infection: the prospective P2C2 HIV study. Journal of Pediatrics, 2002; 141(3): 327-335.

33. STÖBE S, et al. Dynamics in myocardial deformation as an indirect marker of myocardial involvement in acute myocarditis due to HIV infection: a case report. European Heart Journal - Case Reports, 2021; 5(2).

34. SUKRU A, et al. Evolution of Subtle Left Ventricular Systolic Dysfunction by Longitudinal Systolic Strain in Patients with Human Immunodeficiency Virus. Acta Cardiol Sin, 2018; 34: 321-327.

35. UNITED NATIONS PROGRAMME ON HIV/AIDS (UNAIDS). Estatisticas Mundiais sobre o HIV. 2021. Disponível em: https://unaids.org.br/wp-content/uploads/2021/06/2020_11_19_UNAIDS_FactSheet_PORT_Revisada-Final.pdf. Acessado em: 10 out. 2021.

36. VALLILO N, et al. Echocardiographic Follow-up of Perinatally HIV-infected Children and Adolescents: Results from a Single-center Retrospective Cohort Study in Brazil. Pediatr Infect Dis J., 2020; 526-32. 